\title{
Factors determining outcome in gastroschisis: clinical experience over 18 years
}

\author{
L. Cara Jager · Hugo A. Heij
}

Accepted: 3 May 2007 / Published online: 19 June 2007

(C) Springer-Verlag 2007

\begin{abstract}
Although the survival in gastroschisis has improved to more than $85 \%$, serious morbidity with consequent long hospital stay is still very common. Alternative strategies such as planned early delivery and amniotic fluid exchange are being explored. In order to evaluate these approaches, adequate risk stratification of patients with gastroschisis is required. The aim of this study was to identify the risk factors associated with increased morbidity and mortality. A retrospective analysis of all patients with gastroschisis treated at our institution between 1988 and 2005 was conducted. Patients' data were collected from the files and compared for statistical significance with three outcome measures: (1) complicated post-operative course; (2) time to full enteral feeding (TFE); (3) length of hospital stay (LOS). SPSS was used for statistical analysis. Fifty-nine newborns with gastroschisis were admitted during the study period. Antenatal diagnosis was made in 40 cases $(68 \%)$. Associated malformations (other than intestinal anomalies) were present in eight patients. One patient with trisomy 13 was excluded from the analysis. Ten patients (17\%) had compromised bowel (atresia, severe ischaemia or a combination). Primary repair was successful in 47 (81\%) and in 11 patients a silo was applied. Overall survival was 54/58 children (93\%), four patients died. Complications associated with closure occurred in eight patients. Post-
\end{abstract}

L. C. Jager · H. A. Heij $(\bowtie)$

Pediatric Surgical Center of Amsterdam, Emma Children's

Hospital AMC and VU University Medical Center,

P.O. Box 26600, 1100 DD, Amsterdam, The Netherlands

e-mail: H.A.Heij@amc.uva.nl operative course was uncomplicated in 45 patients and complicated in 13. The RR for patients with compromised bowel to have a complicated post-operative course is 9 (95\% CI 2-39) and the RR to die is 20 (95\% CI 2-222) for these patients. Risk stratification of patients with gastroschisis appears possible based on the presence of compromised bowel before closure.

Keywords Gastroschisis - Risk factors - Compromised bowel - Intestinal atresia $\cdot$ Outcome

\section{Introduction}

Although the survival rate in gastroschisis has improved to more than $85 \%$, serious morbidity is still very common. Gastrointestinal complications, intestinal motility and absorption disturbances are frequently present with consequent long and expensive hospital stays [1-4].

Certain patients with gastroschisis seem to be more at risk of a complicated course than others. In several outcome analyses the presence of marked prematurity or intestinal pathology with malrotation, volvulus, infarction, atresia, perforation or stenosis have been recognized as factors associated with adverse outcome [3, 4, 9-11].

To reduce or overcome perinatal morbidity in gastroschisis alternative strategies such as planned early delivery and amniotic fluid exchange are being explored [5-8]. In order to evaluate these approaches, adequate risk stratification of patients with gastroschisis is required. Before antenatal criteria can be identified, postnatal factors will have to be determined.

The aim of this study was to identify the risk factors associated with increased morbidity and mortality in neonates with gastroschisis. 


\section{Materials and methods}

\section{Definitions}

(1) Mortality rate was defined as death during the initial hospital stay. Patients with lethal chromosomal anomalies who were expected to die independent of gastroschisis were excluded from analysis. (2) Complicated post-operative course was defined as the presence of death and/or need for re-intervention, because of closure-related complications or intestinal obstruction. Closure-related complications included abdominal compartment syndrome, ischemia, necrosis, perforation or short bowel syndrome. (3) Time to full enteral feeding (TFE) was defined as the number of days needed to establish full enteral feeding, i.e. $\geq 150 \mathrm{ml} / \mathrm{kg} 24 \mathrm{U}$. (4) Length of hospital stay (LOS) was defined as the number of days between birth and discharge. The child was discharged when full enteral feeding had been achieved and the baby was gaining weight. (5) Compromised bowel was defined as the presence of intestinal atresia, perforation, marked ischemia, necrosis or a combination before intervention.

\section{Study design and patient management}

A retrospective analysis of all neonates with gastroschisis treated at the Pediatric Surgical Center of Amsterdam between 1988 and 2005 was conducted. Patients' data were collected from the files and compared for statistical significance with three outcome measures: (1) complicated post-operative course; (2) time to full enteral feeding (TFE); (3) length of hospital stay (LOS).

Files were reviewed for patient's demographics, prenatal diagnosis, type of delivery, presence of compromised bowel before surgery, silo construction versus primary repair, closure-related complications or intestinal obstruction needing re-intervention, number of days intubated, time to full enteral feeding, minor and major complications, length of hospital stay and survival rate.

Delivery was in our institution if the antenatal diagnosis had been made in one of the affiliated regional hospitals from where neonates were transferred to us for surgical treatment. The obstetric policy was spontaneous vaginal delivery, with caesarean section reserved only on obstetric indication. Immediately after birth the herniated organs were protected to minimize heat and fluid loss. Surgical treatment followed as soon as possible in a hemodynamically stable infant. All procedures were performed in the operating room under general anesthesia. The patients' bowel was carefully inspected for the presence of intestinal atresia or other defects, but as a rule, no attempts were made to correct atresias at the first operation. Primary closure was attempted whenever deemed feasible based on the ease of reduction of abdominal contents as judged by the pediatric surgeon. In the case of excess abdominal tension or compromised ventilation or disproportional abdominal cavity, a delayed closure with placement of a prosthetic silo was performed. Definitive closure of the abdomen was achieved usually within 7 days. In the same surgical session, a tunneled central venous line for parenteral feeding was inserted. TPN was routinely started within $48 \mathrm{~h}$ and continued until establishment of enteral feedings.

\section{Data analysis}

Statistical analysis was performed using SPSS for windows version 13.0. Data were analyzed with the use of Chisquare, Fisher's exact and Mann-Whitney $U$ tests for categorical variables and with the independent sample $t$ test for continuous variables. Kaplan-Meier analysis was performed for length to full enteral feeding and length of hospital stay. Logistic regression analysis was performed to identify independent predictors for adverse outcome and to calculate relative risk factors and $95 \% \mathrm{CI}$ intervals. $P$ values less than 0.05 were considered significant.

\section{Results}

\section{Demographics}

Fifty-nine newborns with gastroschisis were admitted during the study period (Table 1). The mean maternal age was 25 years $(20 \%$ of age $\leq 20$ years) and more than $50 \%$ was primigravida. Gastroschisis was antenatally diagnosed in $68 \%$. Delivery was vaginal in $74 \%$ of the cases. Mean gestational age at birth was 259 days. Fortythree percent of the patients were born preterm ( $<259$ days) of which two patients were borderline premature (222 days). Mean birth weight was 2,490 grams (SE 72). Associated anomalies (except intestinal anomalies) were found in eight patients including hydronephrosis $(n=3)$, patent ductus arteriosus $(n=1)$, tracheomalacia $(n=1)$, undescended testes $(n=1)$, and arthrogryposis of the upper extremity based on a cervical segmental anterior horn dysplasia $(n=1)$. One infant with hypospadia, polydactyly and ventricular septal defect appeared to have the lethal syndrome trisomy 13. Spontaneous death followed after 9 days. He was excluded from further analysis.

Mean time to intervention was $4.3 \mathrm{~h}$. In one case the newborn was transferred by air from another country for surgical treatment in our institution and was successfully operated after almost 2 days.

Primary repair was successful in $47(81 \%)$, and in 11 patients a silo was applied. Median time of mechanical 
Table 1 Characteristics of patients with gastroschisis

\begin{tabular}{|c|c|}
\hline$\overline{\operatorname{Sex}}$ & $35 \mathrm{M} / 24 \mathrm{~F}$ \\
\hline \multirow[t]{2}{*}{ Mean maternal age (years) } & $\begin{array}{l}25(17-35) \\
\quad(20 \% \leq 20)\end{array}$ \\
\hline & $n=40$ \\
\hline \multirow[t]{2}{*}{ Primigravida } & $52.6 \%$ \\
\hline & $\mathrm{n}=51$ \\
\hline Antenatal diagnosis & $40(68 \%)$ \\
\hline Vaginal delivery & $43(74 \%)$ \\
\hline Mean gestational age (days) & $259(222-286)$ \\
\hline Preterm labour (<259 days) & $43 \%$ \\
\hline Mean birth weight $(\mathrm{g})$ & $\begin{array}{l}2,490(1,348- \\
3,720)\end{array}$ \\
\hline Low birth weight $(<2,500 \mathrm{~g})$ & $56 \%$ \\
\hline Associated anomalies & 8 \\
\hline Trisomie 13 & $\begin{array}{l}1 \text { (one death, } \\
\text { excluded) }\end{array}$ \\
\hline Hydronephrosis & 3 \\
\hline Patent ductus arteriosus & 1 \\
\hline Undescended testes & 1 \\
\hline Tracheomalacia & 1 \\
\hline Cervical anterior horn dysplasia & 1 \\
\hline $\begin{array}{l}\text { Compromised bowel condition: severe intestinal } \\
\text { ischemia and/or intestinal atresia }\end{array}$ & $10(18 \%)$ \\
\hline Severe intestinal ischemia only & 2 \\
\hline Intestinal atresia only & 3 \\
\hline Mean time to intervention (h) & 4.3 (SE 0.9) \\
\hline Silo construction & $11(19 \%)$ \\
\hline Median time of ventilation (days) & $5(\mathrm{SE} 1)$ \\
\hline Median time to full enteral feeding, TFE (days) & 32 (SE 3) \\
\hline Median length of hospital stay, LOS (days) & 39 (SE 3) \\
\hline Survival rate & $93 \%$ \\
\hline
\end{tabular}

ventilation was 5 days. Median lengths to enteral and full enteral feeding were, respectively, 15 and 30 days. The median length of hospital stay (LOS) was 39 days.

Ten patients $(17 \%)$ had compromised bowel (severe ischaemia, atresia or a combination), eight of them had intestinal atresia. The post-operative course was uncomplicated in 45 patients $(71 \%)$ and complicated in 13 patients, because of the need for re-intervention and/or death (Table 2).

\section{Complications}

Complications associated with closure occurred in eight patients. All presented a combination of ischemia and/or necrosis and/or perforation of the bowel post-operatively. Four patients developed an abdominal compartment syndrome, of whom three had subsequent perforation of the bowel. Other complications were post-operative anasto-
Table 2 Complications in patients with gastroschisis

\begin{tabular}{ll}
\hline & $n(\%)$ \\
\hline Complicated post-operative course & $13(22 \%)$ \\
Closure-related complications needing & 8 \\
$\quad$ reintervention (s) & \\
Intestinal obstruction needing reintervention (s) & 9 \\
Death & 4 \\
Closure-related complications & In 8 patients \\
& $(14 \%)$ \\
Abdominal compartment syndrome & 4 \\
Ischemia & 4 \\
Necrosis & 6 \\
Perforation & 3 \\
Enterocutaneous fistula & 3 \\
Persistent intestinal obstruction & $9(16 \%)$ \\
Short bowel syndrome & $2(3 \%)$ \\
Mortality & $4(7 \%)$ \\
Bowel resection not compatible with life & $1(+3$ days) \\
Pneumonia, respiratory distress, cardiac asystole & $1(+29$ days $)$ \\
Sudden death after reintervention for persistent & $1(+42$ days $)$ \\
ileus & \\
Persistent shock after operation intestinal & $1(+133$ days $)$ \\
obstruction & \\
cvc sepsis & $22(38 \%)$ \\
Urosepsis & $3(5 \%)$ \\
Meningosepsis & $1(2 \%)$ \\
Pneumonia & $3(5 \%)$ \\
Periventricular leucomalacia & $1(2 \%)$ \\
Inferior vena cava thrombosis & $1(2 \%)$ \\
TPN-related liver function disturbances & $7(12 \%)$ \\
\hline & \\
& \\
&
\end{tabular}

motic leakage $(n=2)$, and enterocutaneous fistulas $(n=3)$. In nine patients re-intervention was needed because of a persistent intestinal obstruction and in four cases caused by intestinal atresia.

Overall survival was 54/58 children (93\%), four patients died. Initially a silo was placed in an infant (GA $37+3$ weeks, BW 2,800 gram) with a compromised bowel condition followed by a laparotomy with jejunal-ileal resection a day later, because of development of necrosis and perforation. After resection the residual bowel length was judged not to be compatible with life and further treatment was withdrawn. The neonate died 3 days after birth. Another patient (GA 37 weeks, BW 2,400 g) without signs of compromised bowel developed pneumonia after primary closure which deteriorated to respiratory distress and cardiac asystole, which eventually did not respond to resuscitation. The third patient (GA $31+5$ weeks, BW $1,390 \mathrm{~g}$ ) presented with a persistent ileus after an initial uncomplicated post-operative course after primary closure, without signs of sepsis or infection. A relaparotomy was 
performed and revealed an atresia not previously identified. An ileocaecal resection was performed. The child died a few days after surgery, 42 days after birth, due to unidentified causes. Autopsy was not permitted. The fourth patient (GA $35+6$, BW 2,420 g) died at the age of 133 days. After birth a possible atresia of the terminal ileum was seen with an abnormal vascular supply. Initially a silo was placed, but development of necrosis necessitated resection and construction of a loop ileostomy. During the operation an intestinal atresia was confirmed with a microcolon. After re-anastomosis of the bowel, intestinal obstruction required another re-intervention. Shortly after this third procedure, the patient died because of persistent shock.

Central venous catheter sepsis occurred in 22 patients. The majority of these periods rapidly responded to antibiotic therapy or catheter removal; cvc sepsis was accompanied by urosepsis in three patients and by meningosepsis in one patient. Other complications were pneumonia $(n=3)$, inferior vena cava thrombosis $(n=1)$, periventricular leukomalacia $(n=1)$ and total parenteral nutritionrelated liver function disturbances $(n=7)$.

\section{Outcome analysis}

The influence of several factors on the outcome parameters: (1) complicated post-operative course (2) time to full enteral feeding (TFE); (3) length of hospital stay (LOS) are shown in Table 3. Preterm delivery, low birth weight, vaginal delivery and type of closure were not significantly different for complicated post-operative course, time to full enteral feeding (TFE) or length of hospital stay (LOS).

Compromised bowel was identified in 10 patients $(18 \%)$. This group had more often significantly a complicated post-operative course characterized by multiple interventions and/or death than those without intestinal atresia/ ischemia $(P=0.005$, Fisher's exact test $)$. Furthermore, the time to establish full enteral feeding and time to discharge were significantly longer in this group

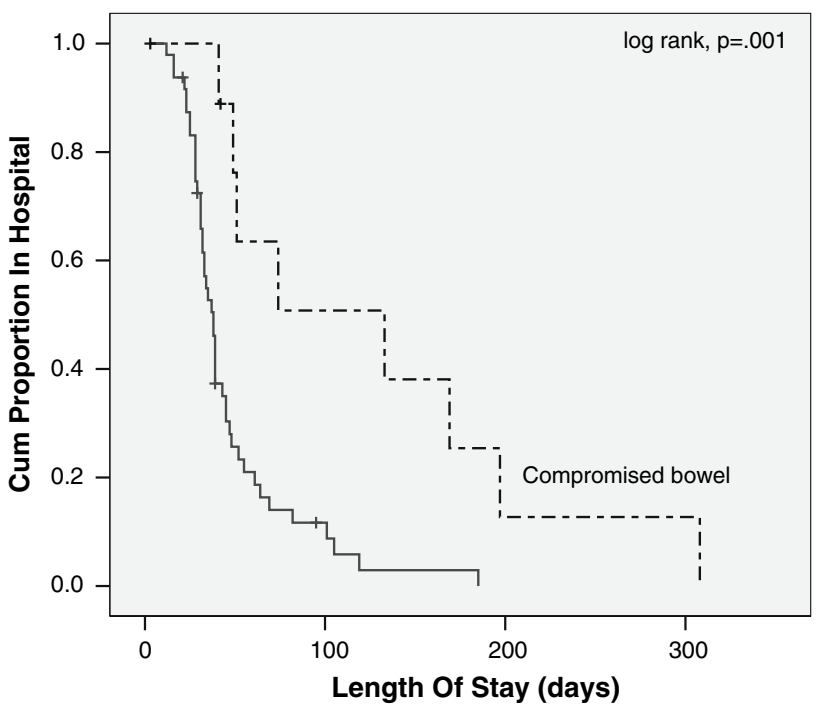

Fig. 1 Kaplan-Meier analysis of gastroschisis patients with and without compromised bowel for length of hospital stay

$(P<0.001, \log$ rank $)$. Figure 1 visualizes a prolonged hospital stay for patients with compromised bowel according to Kaplan-Meier analysis.

Within the compromised bowel group, an intestinal atresia was identified in eight patients. The presence of intestinal atresia was only significantly different for time to full oral feeding and duration of hospital stay $(P<0.006)$.

Sepsis developed in $38 \%$ of the patients, primarily related to central line infections. The group with sepsis significantly more often exhibited a complicated postoperative course $(P=0.020$, Fisher's exact test) and as might be expected the hospital stay was also significantly longer with a delayed time in establishing full enteral feedings $(P<0.001, \log$ rank $)$. Logistic regression analyses were performed for the outcome measures as dependent variables. Compromised bowel and sepsis were both found to be independent predictors for all three outcome measures. No other parameters were found to be predictive. Only compromised bowel was found to be independently

Table 3 Comparison by complicated post operative course (POC), time to full enteral feeding (TFE) and length of hospital stay (LOS)

\begin{tabular}{|c|c|c|c|c|c|c|}
\hline & \multicolumn{2}{|c|}{ Complicated POC ( $n$, patients) } & \multicolumn{2}{|c|}{ Time TFE (median, days) } & \multicolumn{2}{|c|}{ LOS (median, days) } \\
\hline & Yes/No & $P$ value & Yes/No & $P$ value & Yes/No & $P$ value \\
\hline Vaginal delivery & $8 / 5$ & 0.294 & $30 / 39$ & 0.727 & $38 / 45$ & 0.674 \\
\hline Preterm labour & $4 / 9$ & 0.350 & $32 / 30$ & 0.550 & $41 / 39$ & 0.698 \\
\hline Low birth weight & $6 / 7$ & 0.528 & $32 / 25$ & 0.988 & $43 / 34$ & 0.957 \\
\hline Primary closure & $8 / 5$ & 0.101 & $30 / 45$ & 0.053 & $37 / 55$ & 0.090 \\
\hline Compromised bowel & $6 / 7$ & 0.005 & $150 / 29$ & 0.000 & $133 / 38$ & 0.001 \\
\hline Intestinal atresia & $4 / 4$ & 0.066 & $68 / 30$ & 0.002 & $74 / 38$ & 0.006 \\
\hline cvc Sepsis & $9 / 4$ & 0.020 & $43 / 26$ & 0.001 & $52 / 35$ & 0.000 \\
\hline
\end{tabular}


associated with increased mortality $(P=0.013)$. The relative risk for patients with compromised bowel to have a complicated post-operative course is 9 (95\% CI 2-39, $P=0.004)$ and the relative risk to die is 20 (95\% CI 2-222, $P=0.014$ ) for these patients.

The RR for a neonate with a compromised bowel to have a more prolonged hospital stay than a neonate without intestinal defects is 4.7 (95\% CI $1.8-11.7, P<0.001)$; the RR for a longer duration of hospital stay with the presence of sepsis is 3.6 (95\% CI $1.8-7.2, P<0.001)$.

\section{Discussion}

Similar to other studies [1-4, 9-11] the overall survival rate of patients with gastroschisis in this cohort was $93 \%$ (54 of 58). Although this survival in gastroschisis is fairly good gastrointestinal complications, intestinal motility and absorption disturbances occurred frequently with consequent long and expensive hospital stays [1-4]. In our series $22 \%$, approximately one out of five patients with gastroschisis, was jeopardized with a complicated post-operative course, characterized by multiple interventions, possible death and if surviving with a significant increased time to establish oral feedings and prolonged hospital stay.

The degree of gastrointestinal compromise after birth and the impact on outcome has been subject of only few studies; in most papers the presence of intestinal atresia has been compared to outcome. In our experience intestinal atresia is often not a solitary occurrence but accompanied by intestinal abnormalities. To determine whether presence of intestinal pathology influences outcome, we defined compromised bowel as the presence of intestinal atresia, severe ischemia, necrosis, volvulus, perforation or a combination. In the literature several other factors have also been found to be associated with adverse outcome, including marked prematurity, mode of delivery and method of closure [3, 4, 9-11].

After regression analysis of these possible risk factors we demonstrated that presence of compromised bowel was associated with a need for significantly more surgical procedures (complicated post-operative course), with a delayed establishment of full enteral feeding and with a prolonged hospital stay. Also a significant difference in mortality rate was observed if compromised bowel was present. This concurs with the findings of Molik et al. [9] who categorized their series of 103 neonates born with gastroschisis into a simple and complex group by the presence of atresia, stenosis, perforation or ischemia and compared the groups for outcome. They also found a strikingly significant difference in mortality rate; 9 out of 32 patients with a complex bowel condition $(n=26$ atresias) died versus $0 \%$ in the simple group. Complex cases also had a longer time delay before establishing full enteral feedings and a three-times longer hospitalization period.

Other studies on gastroschisis also report increased mortality and morbidity of gastroschisis in the presence of intestinal atresia [3, 4, 12]. In our cohort the majority of the patients with compromised bowel presented with intestinal atresia (8/10). Intestinal atresia is therefore an important risk factor, probably due to the dilated bowel that causes increased abdominal pressure and interferes with closure and placement of silo.

A large outcome analysis of 185 patients showed that 18 of $21(86 \%)$ of patients with intestinal atresia survived. Although patients with intestinal atresia had a significantly prolonged hospital stay, no correlation with morbidity and mortality could be demonstrated in that study. Only intestinal necrosis was an independent predictor of increased mortality [10]. Driver et al. [4] also demonstrated a significant difference for patients with intestinal atresia in a prolonged length of hospital stay, but concluded that the presence of intestinal atresia alone was not predictive of increased mortality. Baerg et al. [3] concluded that both the presence of intestinal atresia as well as development of intestinal necrosis were significantly associated with mortality.

Our regression analysis also demonstrated that sepsis was significantly more frequent in the complicated postoperative group and time to full enteral feeding and the length of hospital stay were significantly longer, butdid not correlate with mortality. We explain the higher incidence of cvc sepsis primarily by the longer need for parenteral nutrition until full enteral feeding is reached, which is the case in patients with a more complicated post-operative course. The possible role of early commencement of trophic feeds on the incidence of sepsis was not investigated in this study due to retrospective limitations.

Vaginal delivery did not correlate with adverse outcome in this study. This has been confirmed in other published reports, in which either no benefit to routine caesarean section or an adverse outcome with vaginal delivery was shown [11-14]. As there is no clear benefit of elective caesarean section over vaginal delivery, in our opinion the decision should only be guided by obstetrical indications.

Based on the hypothesis that prolonged exposure of the fetal gut to amniotic fluid is a significant factor in the development of the typical bowel damage [6-8, 15], much debate has taken place regarding the possible benefit of preterm delivery. On the other hand, prematurity and low birth weight seem to correlate with adverse outcome, such as higher occurrence of sepsis and longer time to full feeds and hospitalization period [1, 10, 16]. However, in our analysis low birth weight and preterm labor were not associated with adverse outcome. A prospective trial of elective preterm delivery using specific ultrasound criteria 
resulted in improved surgical outcome without significant morbidity secondary to prematurity [5].

No difference in outcome was observed in the current study between primary or staged closure. In the case of excess abdominal tension due to disproportional abdominal cavity or compromised ventilation, delayed closure with placement of a prosthetic silo was performed ( $n=11$, of which 3 with compromised bowel, ns). The treatment of choice for gastroschisis has been extensively debated and literature presents a great heterogeneity among the available evidence [17-21]. Closure-related complications after primary closure could be minimized with the use of more objective measurements for excessive intra-abdominal pressure peri-operatively [22, 23]. According to Olesevich et al. [23], measuring the intra operative bladder pressure as parameter for intra- abdominal pressure improves the safety of primary closure by avoiding bowel ischemia and leads to a shorter time to feeds and reduced length of hospital stay.

In summary, our study underlines that overall prognosis for a gastroschisis patient is good. Risk stratification of patients with gastroschisis appears possible, based on the presence of atresia and/or other intestinal damage before closure. Compromised bowel is the main predictor of a complicated post-operative course with a longer time delay in establishment of full enteral feeding and length of hospital stay. To reduce or overcome perinatal morbidity in gastroschisis alternative strategies are being explored, like planned early delivery, intra- abdominal pressure measurement and amniotic fluid exchange [5-8, 23, 24]. For a proper evaluation of these new strategies, it is important that patients are stratified according to risk. Our data contribute to this risk stratification as it identifies the compromised bowel condition as the main determining factor for postnatal complications. The next step will be to investigate whether there is a similar correlation between the condition of the antenatal bowel and outcome.

\section{References}

1. Wilson RD, Johnson MP (2004) Congenital abdominal wall defects: an update. Fetal Diagn Ther 19:385-398

2. Hunter A, Soothill Peter (2002) Gastroschisis-an overview. Prenat Diagn 22:869-873

3. Baerg J, Kaban G, Pahwa TP, Reid D (2003) Gastroschisis: a sixteen- year review. J Pediatr Surg 38:771-774

4. Driver CP, Bruce J, Bianchi A et al (2000) The contemporary outcome of gastroschisis. J Pediatr Surg 35:1719-1723
5. Moir CR, Ramsey PS, Ogburn PL, Johnson RV, Ramin KD (2004) A prospective trial of elective preterm delivery for fetal gastroschisis. Am J Perinatol 21(5):289-294

6. Luton D (1999) Effect of amnio- infusion on the outcome of prenatally diagnosed gastroschisis. Fetal Diagn Ther 14(3):152155

7. Aktug $T$, et al (2001) Amnioallantoic fluid exchange for the prevention of intestinal damage in gastroschisis III: determination of the waste products removed by exchange. Eur J Pediatr Surg Int 17:116-119

8. Correia- Pinto J, Tavares LL, Baptista MJ et al (2002) Meconium dependence of bowel damage in gastroschisis. J Ped Surg 37:3135

9. Molik KA, Gingalewski CA, West KW et al (2001) Gastroschisis: a plea for risk categorization. J Pediatr Surg 36:51-55

10. Snyder CL (1999) Outcome analysis for gastroschisis. J Pediatr Surg 34:1253-1256

11. Singh SJ, Fraser A, Leditschke JF et al (2003) Gastroschisis: determinants of neonatal outcome. Pediatr Surg Int 19:260-265

12. Salihu HM, Emusu D, Aliyu Z et al (2004) Mode of delivery and neonatal survival of infants with isolated gastroschisis. Obstet Gynecol 104:678-683

13. Puligandla PS, Janvier A, Flageole H et al (2004) Routine cesarean delivery does not improve the outcome of infants with gastroschisis. J Ped Surg 39:742-745

14. Segel SY, Marder SJ, et al (2001) Fetal abdominal defects and mode of dlivery: a systematic review. Obstet Gynecol 98:867-873

15. Bittencourt DG, et al (2006) Impact of corticosteroid on intestinal injury in a gastroschisis rat model: morphometric analysis. J Pediatr Surg 41:547-553

16. Ergün O, Barksdale E, FS Ergün et al (2005) The timing of delivery of infants with gastroschisis influences outcome. Independent report. Elsevier, Amsterdam

17. Davies MW, Kimble RM, Woodgate PG (2002) Ward reduction without general anaesthesia versus reduction and repair under general anesthesia for gastroschisis in newborn infants. Cochrane Database Syst Rev, issue 3

18. Bianchi A, Dickson AP, Alizai NK (2002) Elective delayed midgut reduction-no anesthesia for gastroschisis: selection and conversion criteria. J Pediatr Surg 37(9):1334-1336

19. Schlatter M, Norris K, Uitvlugt $N$ et al (2003) Improved outcomes in the treatment of gastroschisis using a preformed silo and delayed repair approach. J Pediatr Surg 38:459-464

20. Fleet MS, Hunt de la MN (2000) Intestinal atresia with gastroschisis: a selective approach to management. J Pediatr Surg 35:1323-1325

21. Hoehner JC, Ein SH, Kim PCW (1998) Management of gastroschisis with concomitant jejuno-ileal atresia. J Pediatr Surg 33:885-888

22. McGuigan RM, Azarow KS (2004) Is splanchnic perfusion pressure more predictive of outcome than intragastric pressure in neonates with gastroschisis? Am J Surg 187:609-611

23. Olesevich M, Alexander F, Khan M, Cotman K (2005) Gastroschisis revisited: role of intraoperative measurement of abdominal pressure. J Pediatr Surg 40:789-792

24. Vegunta RK, Wallace LJ, Leonardi MR, Gross TL et al (2005) Perinatal management of gastroschisis: analysis of a newly established clinical pathway. J Pediatr Surg 40:528-534 